\title{
Filtering design for two-dimensional Markovian jump systems with state- delays and deficient mode information ${ }^{\text {is }}$
}

\author{
Yanling Wei ${ }^{\text {a }}$, Jianbin Qiu ${ }^{\mathrm{a}, *}$, Hamid Reza Karimi ${ }^{\mathrm{b}}$, Mao Wang ${ }^{\mathrm{a}}$
}

\author{
${ }^{a}$ Research Institute of Intelligent Control and Systems, Harbin Institute of Technology, Harbin 150001, PR China ${ }^{\mathrm{b}}$ Department of Engineering, Faculty of \\ Engineering and Science, University of Agder, N-4898 Grimstad, Norway
}

\author{
Keywords: \\ Two-dimensional system \\ Markovian jump system \\ $\mathcal{H}_{\infty}$ filtering \\ Deficient mode information \\ State-delay
}

\begin{abstract}
A B S T R A C T
This paper is concerned with the problem of $\mathcal{H}_{\infty}$ filtering for a class of two-dimensional Markovian jump linear systems described by the Fornasini-Marchesini local state-space model. The systems under consideration are subject to state-delays and deficient mode information in the Markov chain. The description of deficient mode information is compre-hensive that simultaneously includes the exactly known, partially unknown and uncertain transition probabilities. By invoking the properties of the transition probability matrix, together with the convexification of uncertain domains, a new $\mathcal{H}_{\infty}$ performance analysis criterion for the filtering error system is firstly derived. Then, via some matrix inequality linearisation procedures, two approaches for the filter synthesis are proposed. It is shown that both the full-order and reduced-order filters can be constructed by solving a set of lin-ear matrix inequalities. Finally, simulation studies are provided to illustrate the effective-ness of the proposed design methods.
\end{abstract}

\section{Introduction}

It is well known that many physical processes or systems have intrinsic multi-dimensional (m-D) characteristics [14]. Among various m-D systems, in particular the two-dimensional (2-D) systems have received considerable research interests due to their wide applications in different areas, such as 2-D digital filtering [17,33], linear image processing [23,32], and repetitive processes control [7,41]. In recent years, a great number of significant results on analysis and synthesis of 2-D systems have been reported in the open literature. To mention a few, the stability problem for 2-D systems was investigated in $[5,10]$. The control and filtering problems were considered in $[6,8,18,30,33,35]$. The model approximation problem was addressed in $[32,40]$.

On the other hand, Markovian jump linear systems (MJLSs), initially introduced by Krasovskii and Lidskii [13], have been widely investigated in the past decades, and a great deal of elegant results have been obtained $[1,4,9,11,15,16,22,24-$ $26,31,34,36,38,42]$. The motivation for the study of this class of systems is the fact that many dynamical systems subject to random abrupt variations can be modeled by MJLSs, which are described by a set of classical differential (or difference) equations and a Markov stochastic process (or Markov chain) [2]. It is known that 2-D systems are no exception for this case. As a critical factor, the transition probabilities (TPs) in the Markov chain determine the system behavior and performance, and many analysis and synthesis issues on MJLSs have been exploited assuming the perfect information on the TPs. However,

\footnotetext{
th This work was supported in part by the National Natural Science Foundation of China (61374031, 61333012), the Program for New Century Excellent Talents in University (NCET-12-0147), the State Key Laboratory of Robotics and Systems (HIT), and the Alexander von Humboldt Foundation of Germany. * Corresponding author.

E-mail addresses: yanlingwei@hit.edu.cn (Y. Wei), jbqiu@hit.edu.cn (J. Qiu), hamid.r.karimi@uia.no (H.R. Karimi), maowang@hit.edu.cn (M. Wang).
} 
in many practical scenarios, it is questionable to obtain all the precise mode transition information, and the cost is probably high. Thus, it is significant and challenging, from control perspectives, to further study more general jump systems with deficient mode information $[2,12,37,39,43,44]$. Specifically, the authors in [39] addressed the robust $\mathcal{H}_{\infty}$ filtering problem for a class of MJLSs with norm-bounded uncertain TPs. The authors in [43] investigated the $\mathcal{H}_{\infty}$ filtering problem for a class of MJLSs with partially unknown TPs. Unfortunately, the aforementioned results are only concerned with one-dimensional (1D) systems. In addition, it has also been well recognised that time-delay, which is frequently a source of instability and performance degradation, exists commonly in MJLSs [11,27-29,38]. However, to the authors' best knowledge, few re-sults have been reported on the $\mathcal{H}_{\infty}$ filtering for 2-D MJLSs with state-delays and deficient mode information, which simultaneously involves the exactly known, partially unknown and uncertain TPs. This motivates us for the present study.

In this paper, we will tackle the $\mathcal{H}_{\infty}$ filtering problem for a class of 2-D MJLSs described by the Fornasini-Marchesini local state-space model. The systems under consideration are subject to state-delays and deficient mode information, which simultaneously incorporates the exactly known, partially unknown and uncertain TPs. By the properties of the transition probability matrix and the convexification of uncertain domains, a new sufficient condition for $\mathcal{H}_{\infty}$ performance analysis of filtering error system will be firstly presented. Then via some matrix inequality linearisation procedures, two approaches for the filter synthesis will be proposed. It is shown that both the full-order and reduced-order filters can be obtained by solving a set of linear matrix inequalities. Simulation studies will be finally performed to show the effectiveness of the pro-posed filtering design methods. Compared with the existing results on robust $\mathcal{H}_{\infty}$ filtering for 2-D discrete-time MJLSs with statedelays, the main contributions of the work in this paper are twofold: (i) The filtering problem for a class of 2-D discrete-time MJLSs with deficient mode information, which simultaneously includes the exactly known, partially unknown, and polytopic-type uncertain TPs, is considered. The corresponding filtering design results are expected to be more general and thereby more practicable. (ii) By a linearisation approach, together with Projection lemma, some less conservative filter synthesis conditions are obtained for 2-D MJLSs with state-delays.

Notations. The notations used throughout the paper are standard. $\mathbf{R}^{n}$ denotes the $n$-dimensional Euclidean space; $\operatorname{Sym}\{A\}$ is the shorthand notation for $A+A^{\mathrm{T}}$; the notation $P>0$ means that $P$ is real symmetric and positive definite; $\mathbf{I}$ and $\mathbf{0}$ repre-sent the identity matrix and a zero matrix, respectively; $(\mathcal{S}, \mathcal{F}, \mathcal{P})$ denotes a complete probability space, in which $\mathcal{S}$ is the sample space, $\mathcal{F}$ is the $\sigma$ algebra of subsets of the sample space, and $\mathcal{P}$ is the probability measure on $\mathcal{F} ; \mathbb{E}[\cdot]$ stands for the mathematical expectation;|| $\|$ refers to the Euclidean norm of a vector or its induced norm of a matrix; $l_{2}\{[0, \infty),[0, \infty)\}$ denotes the space of square summable sequences on $\{[0, \infty),[0, \infty)\}$. Matrices, if not explicitly stated, are assumed to have appropriate dimensions for algebra operations.

\section{Problem formulation and preliminaries}

Fix a complete probability space $(\mathcal{S}, \mathcal{F}, \mathcal{P})$ and consider the following two-dimensional (2-D) discrete-time Markovian jump linear systems (MJLSs) represented by the Fornasini-Marchesini (FM) local state-space (LSS) model with state-delays in each of the two independent directions of information propagation,

$$
\begin{aligned}
&(\Sigma): x(i+1, j+1)= A_{1}(r(i, j+1)) x(i, j+1)+A_{2}(r(i+1, j)) x(i+1, j) \\
&+A_{d 1}(r(i, j+1)) x\left(i-d_{1}, j+1\right)+A_{d 2}(r(i+1, j)) x\left(i+1, j-d_{2}\right) \\
&+B_{1}(r(i, j+1)) w(i, j+1)+B_{2}(r(i+1, j)) w(i+1, j), \\
& y(i, j)=C(r(i, j)) x(i, j)+D(r(i, j)) w(i, j), \\
& z(i, j)=L(r(i, j)) x(i, j),
\end{aligned}
$$

where $x(i, j) \in \mathbf{R}^{n_{x}}$ is the state vector; $y(i, j) \in \mathbf{R}^{n_{y}}$ is the measured output; $z(i, j) \in \mathbf{R}^{n_{z}}$ is the objective signal to be estimated; $w(i, j) \in \mathbf{R}^{n_{w}}$ denotes the disturbance input vector which belongs to $l_{2}\{[0, \infty),[0, \infty)\}$; and $d_{1}$ and $d_{2}$ are two constant positive integers representing delays along vertical and horizontal directions, respectively. $A_{1}(r(i, j+1)), A_{2}(r(i+1, j)), A_{d 1}(r(i, j+1))$, $A_{d 2}(r(i+1, j)), B_{1}(r(i, j+1)), B_{2}(r(i+1, j)), C(r(i, j)), D(r(i, j))$, and $L(r(i, j))$ are real-valued system matrices. These matrices are functions of $r(i, j)$, which is described by a discrete-time, discrete-state homogeneous Markov chain with a finite state-space $\mathcal{I}:=\{1, \ldots, N\}$ and a stationary transition probability matrix (TPM) $\Pi=\left[\pi_{m n}\right]_{N \times N}$, where

$$
\pi_{m n}=\operatorname{Pr}(r(i+1, j+1)=n \mid r(i, j+1)=m)=\operatorname{Pr}(r(i+1, j+1)=n \mid r(i+1, j)=m), \quad \forall m, n \in \mathcal{I},
$$

with $\pi_{m n} \geqslant 0$ and $\sum_{n=1}^{N} \pi_{m n}=1$. For $r(i, j)=m \in \mathcal{I}$, the system matrices of the $m$ th mode are denoted by $\left(A_{1 m}, A_{2 m}, A_{d 1 m}, A_{d 2 m}, B_{1 m}, B_{2 m}, C_{m}, D_{m}, L_{m}\right)$, which are real known and with appropriate dimensions. Unless otherwise stated, similar simplification is also applied to other matrices in the following.

Remark 1. The unique characteristic of 2-D process is that the information propagation occurs in each of the two independent directions. Attempts to analyse these processes using traditional one-dimensional (1-D) systems theory fail (except for a few very restrictive special cases) because such an approach ignores their inherent 2-D systems structure. For instance, when 1-D Markov chains are employed to model a gas absorption with Markovian jump parameters, the state evolution for the underlying systems can be represented by $x(i+1)=A(r(i)) x(i)+A_{d}(r(i)) x\left(i-d_{1}\right)+B_{1}(r(i)) w(i)$. Alternatively, by utilising the 2-D MJLSs to describe the above scenarios, the Markovian dynamic model can be expressed 
as FM LSS model (1). Since $x(i+1, j+1)$ in (1) can be viewed as the one-step forward shift from $x(i, j+1)$ in the direction of $i$ coordinate or $x(i+1, j)$ in the direction of $j$ coordinate, we need two vectors to identify the relationship among $x(i+1, j+1)$, $x(i, j+1)$ and $x(i+1, j)$, which can describe the information propagation more accurately. This can also well explain that such a 2-D framework leads to more complex structure than 1-D case, and it is thus more challenging and difficult to investigate the analysis and synthesis of 2-D systems.

In this paper, the transition probabilities (TPs) of the jumping process are assumed to be uncertain and partially accessed, i.e., the TPM $\Pi=\left[\pi_{m n}\right]_{N \times N}$ is assumed to belong to a given polytope $P_{\Pi}$ with vertices $\Pi_{s}, s=1,2, \ldots, M$, $P_{\Pi}:=\left\{\Pi \mid \Pi=\sum_{s=1}^{M} \alpha_{s} \Pi_{s} ; \alpha_{s} \geqslant 0, \sum_{s=1}^{M} \alpha_{s}=1\right\}$, where $\Pi_{s}=\left[\pi_{m n}\right]_{N \times N}, m, n \in \mathcal{I}$, are given TPMs containing unknown elements still. For instance, for system $(\Sigma)$ with four operation modes, the TPM may be as,

$$
\left[\begin{array}{llll}
\pi_{11} & \tilde{\pi}_{12} & \hat{\pi}_{13} & \pi_{14} \\
\hat{\pi}_{21} & \pi_{22} & \tilde{\pi}_{23} & \pi_{24} \\
\pi_{31} & \hat{\pi}_{32} & \pi_{33} & \hat{\pi}_{34} \\
\pi_{41} & \tilde{\pi}_{42} & \hat{\pi}_{43} & \hat{\pi}_{44}
\end{array}\right],
$$

where the elements labeled with “^” and “ ” represent the unknown information and polytopic uncertainties on TPs, respectively, and the others are known TPs. For notational clarity, $\forall m \in \mathcal{I}$, we denote $\mathcal{I}=\mathcal{I}_{\mathcal{K}}^{(m)} \cup \mathcal{I}_{\mathcal{U C}}^{(m)} \cup \mathcal{I}_{\mathcal{U K}}^{(m)}$ as follows,

$$
\begin{aligned}
& \mathcal{I}_{\mathcal{K}}^{(m)}:=\left\{n: \pi_{m n} \text { is known }\right\}, \\
& \mathcal{I}_{\mathcal{U C}}^{(m)}:=\left\{n: \tilde{\pi}_{m n} \text { is uncertain }\right\}, \\
& \mathcal{I}_{\mathcal{U K}}^{(m)}:=\left\{n: \hat{\pi}_{m n} \text { is unknown }\right\} .
\end{aligned}
$$

Also, we denote

$$
\pi_{\mathcal{U}}^{(m s)}:=\sum_{n \in \mathcal{I}_{\mathcal{L}}^{(m)}} \hat{\pi}_{m n}=1-\sum_{n \in \mathcal{I}_{\mathcal{K}}^{(m)}} \pi_{m n}-\sum_{n \in \mathcal{I}_{\mathcal{U C}}^{(m)}} \tilde{\pi}_{m n}^{(s)},
$$

where $\tilde{\pi}_{m n}^{(s)}$ represents an uncertain TP in the sth polytope, $\forall s=1, \ldots, M$.

The boundary conditions of system $(\Sigma)$ in (1) are given by,

$$
\begin{aligned}
& \left\{x(i, j)=\phi(i, j), \quad \forall j \geqslant 0, \quad-d_{1} \leqslant i \leqslant 0\right\} ; \\
& \left\{x(i, j)=\varphi(i, j), \quad \forall i \geqslant 0, \quad-d_{2} \leqslant j \leqslant 0\right\} ; \\
& \phi(0,0)=\varphi(0,0) .
\end{aligned}
$$

Throughout this paper, the following assumptions are made.

Assumption 1. System $(\Sigma)$ in $(1)$ is stochastically stable.

Assumption 2. The boundary condition is assumed to satisfy

$$
\lim _{T_{1} \rightarrow \infty} \mathbb{E}\left\{\sum_{j=0}^{T_{1}} \sum_{i=-d_{1}}^{0}\left(\phi^{\mathrm{T}}(i, j) \phi(i, j)\right)\right\}+\lim _{T_{2} \rightarrow \infty} \mathbb{E}\left\{\sum_{i=0}^{T_{2}} \sum_{j=-d_{2}}^{0}\left(\varphi^{\mathrm{T}}(i, j) \varphi(i, j)\right)\right\}<\infty .
$$

The aim of the $\mathcal{H}_{\infty}$ filtering problem to be addressed in this paper is to estimate the signal $z(i, j)$ by a linear dynamic filter of the following mode-dependent FM-type structure,

$$
\begin{aligned}
\hat{x}(i+1, j+1)= & A_{f 1}(r(i, j+1)) \hat{x}(i, j+1)+A_{f 2}(r(i+1, j)) \hat{x}(i+1, j) \\
& +B_{f 1}(r(i, j+1)) y(i, j+1)+B_{f 2}(r(i+1, j)) y(i+1, j), \\
\hat{z}(i, j)=C_{f}(r(i, j)) \hat{x}(i, j)+D_{f}(r(i, j)) y(i, j), & \text { for } i=0 \quad \text { or } j=0,
\end{aligned}
$$

where $\hat{x}(i, j) \in \mathbf{R}^{n_{f}}$ is the filter state; $\hat{z}(i, j)$ is the estimation of $z(i, j)$; and $A_{f 1}(r(i, j+1)) \in \mathbf{R}^{n_{f} \times n_{f}}, A_{f 2}(r(i+1, j)) \in \mathbf{R}^{n_{f} \times n_{f}}$, $B_{f 1}(r(i, j+1)) \in \mathbf{R}^{n_{f} \times n_{y}}, B_{f 2}(r(i+1, j)) \in \mathbf{R}^{n_{f} \times n_{y}}, C_{f}(r(i, j)) \in \mathbf{R}^{n_{2} \times n_{f}}$, and $D_{f}(r(i, j)) \in \mathbf{R}^{n_{z} \times n_{y}}$ are mode-dependent filter gains to be determined. It is noted that in this paper, we consider both the full-order and reduced-order filters, that is, $n_{f}=n_{x}$ for the full-order filter and $n_{f}<n_{x}$ for the reduced-order filter.

Augmenting the model in (1) to include the states of the filter in (5), we obtain the following 2-D filtering error system,

$$
\begin{aligned}
(\bar{\Sigma}): \bar{x}(i+1, j+1)= & \bar{A}_{1}(r(i, j+1)) \bar{x}(i, j+1)+\bar{A}_{2}(r(i+1, j)) \bar{x}(i+1, j) \\
& +\bar{A}_{d 1}(r(i, j+1)) E \bar{x}\left(i-d_{1}, j+1\right)+\bar{A}_{d 2}(r(i+1, j)) E \bar{x}\left(i+1, j-d_{2}\right) \\
& +\bar{B}_{1}(r(i, j+1)) w(i, j+1)+\bar{B}_{2}(r(i+1, j)) w(i+1, j), \\
\bar{z}(i, j)=\bar{C}(r(i, j)) \bar{x}(i, j) & +\bar{D}(r(i, j)) w(i, j),
\end{aligned}
$$


where $\bar{x}(i, j):=\left[\begin{array}{ll}x^{\mathrm{T}}(i, j) & \hat{x}^{\mathrm{T}}(i, j)\end{array}\right]^{\mathrm{T}}, \bar{z}(i, j):=z(i, j)-\hat{z}(i, j)$, and

$$
\begin{aligned}
& \bar{A}_{1}(r(i, j+1)):=\left[\begin{array}{cc}
A_{1}(r(i, j+1)) & \mathbf{0} \\
B_{f 1}(r(i, j+1)) C(r(i, j+1)) & A_{f 1}(r(i, j+1))
\end{array}\right], \\
& \bar{A}_{2}(r(i+1, j)):=\left[\begin{array}{cc}
A_{2}(r(i+1, j)) & \mathbf{0} \\
B_{f 2}(r(i+1, j)) C(r(i+1, j)) & A_{f 2}(r(i+1, j))
\end{array}\right], \\
& \bar{A}_{d 1}(r(i, j+1)):=\left[\begin{array}{c}
A_{d 1}(r(i, j+1)) \\
\mathbf{0}
\end{array}\right], \quad \bar{A}_{d 2}(r(i+1, j)):=\left[\begin{array}{c}
A_{d 2}(r(i+1, j)) \\
\mathbf{0}
\end{array}\right], \\
& \bar{B}_{1}(r(i, j+1)):=\left[\begin{array}{c}
B_{1}(r(i, j+1)) \\
B_{f 1}(r(i, j+1)) D(r(i, j+1))
\end{array}\right], \\
& \bar{B}_{2}(r(i+1, j)):=\left[\begin{array}{c}
B_{2}(r(i+1, j)) \\
B_{f 2}(r(i+1, j)) D(r(i+1, j))
\end{array}\right], \\
& \bar{C}(r(i, j)):=\left[L(r(i, j))-D_{f}(r(i, j)) C(r(i, j)) \quad-C_{f}(r(i, j))\right], \\
& \bar{D}(r(i, j)):=-D_{f}(r(i, j)) D(r(i, j)), \quad E:=\left[\begin{array}{ll}
\mathbf{I}_{n_{x}} & \mathbf{0}_{n_{x} \times n_{f}}
\end{array}\right] .
\end{aligned}
$$

Now, for a more precise description of the main objective of this paper, we introduce the following definitions.

Definition $1([14,33])$. The filtering error system $(\bar{\Sigma})$ in $(6)$ is said to be stochastically stable if for $w(i, j)=0$ and the boundary condition satisfying (4), the following condition holds,

$$
\mathbb{E}\left\{\sum_{i=0}^{\infty} \sum_{j=0}^{\infty}\left(\|\bar{x}(i, j+1)\|^{2}+\|\bar{x}(i+1, j)\|^{2}\right)\right\}<\infty .
$$

Definition 2 ([14,33]). Given a scalar $\gamma>0$, system $(\bar{\Sigma})$ in (6) is said to be stochastically stable with an $\mathcal{H}_{\infty}$ disturbance attenuation performance index $\gamma$ if it is stochastically stable with $w(i, j)=0$, and under zero boundary conditions $\phi(i$, $j)=\varphi(i, j)=0$ in $(3)$, satisfies

$$
\|\tilde{z}\|_{\mathbb{E}_{2}}<\gamma\|\tilde{w}\|_{2},
$$

where

$$
\begin{aligned}
& \|\tilde{z}\|_{\mathbb{E}_{2}}:=\sqrt{\mathbb{E}\left\{\sum_{i=0}^{\infty} \sum_{j=0}^{\infty}\left(\|\bar{z}(i, j+1)\|^{2}+\|\bar{z}(i+1, j)\|^{2}\right)\right\}}, \\
& \|\tilde{w}\|_{2}:=\sqrt{\sum_{i=0}^{\infty} \sum_{j=0}^{\infty}\left(\|w(i, j+1)\|^{2}+\|w(i+1, j)\|^{2}\right)},
\end{aligned}
$$

for all non-zero $w \in l_{2}\{[0, \infty),[0, \infty)\}$ and under zero boundary conditions.

Therefore, the objective of this paper is to design an $\mathcal{H}_{\infty}$ filter in the form of (5), such that the 2-D filtering error system $(\bar{\Sigma})$ in $(6)$ with deficient mode information is stochastically stable with a prescribed $\mathcal{H}_{\infty}$ performance index $\gamma$.

\section{Main results}

In this section, based on a Markovian Lyapunov-Krasovskii functional (MLKF), a new $\mathcal{H}_{\infty}$ performance analysis criterion for the two-dimensional (2-D) filtering error system $(\bar{\Sigma})$ in $(6)$ with deficient mode information will be firstly derived. Then, by some matrix inequality linearisation techniques, two approaches to the $\mathcal{H}_{\infty}$ filter synthesis will be developed.

\section{1. $\mathcal{H}_{\infty}$ filtering analysis}

In this subsection, by the properties of the transition probability matrix (TPM) and the convexification of uncertain domains, an $\mathcal{H}_{\infty}$ performance analysis criterion for the 2-D filtering error system $(\bar{\Sigma})$ in (6) with deficient mode information is presented, which will play a key role in solving the $\mathcal{H}_{\infty}$ filter synthesis problem.

Proposition 1. The 2-D MJLS with state-delays and deficient mode information in (6) is stochastically stable with a guaranteed $\mathcal{H}_{\infty}$ performance $\gamma$, if there exist positive-definite symmetric matrices $\left\{P_{1 m}, P_{2 m}\right\} \in \mathbf{R}^{\left(n_{x}+n_{f}\right) \times\left(n_{x}+n_{f}\right)}$, and $\left\{Q_{1}, Q_{2}\right\} \in \mathbf{R}^{n_{x} \times n_{x}}$, such that the following matrix inequalities hold, 


$$
\mathcal{A}_{m}^{\mathrm{T}} \mathcal{P}_{n}^{(s)} \mathcal{A}_{m}+\mathcal{L}_{m}^{\mathrm{T}} \mathcal{L}_{m}+\Theta_{m}<0, \quad m \in \mathcal{I}, \quad n \in \mathcal{I}_{\mathcal{U} \mathcal{K}}^{(m)}, \quad s=1, \ldots, M,
$$

where

$$
\begin{aligned}
& \Theta_{m}:=\operatorname{diag}\left\{-P_{1 m}+E^{\mathrm{T}} Q_{1} E,-P_{2 m}+E^{\mathrm{T}} Q_{2} E,-Q_{1},-Q_{2},-\gamma^{2} I,-\gamma^{2} I\right\}, \\
& \mathcal{A}_{m}:=\left[\begin{array}{llllll}
\bar{A}_{1 m} & \bar{A}_{2 m} & \bar{A}_{d 1 m} & \bar{A}_{d 2 m} & \bar{B}_{1 m} & \bar{B}_{2 m}
\end{array}\right], \quad E:=\left[\begin{array}{lll}
I_{n_{x}} & 0_{n_{x} \times n_{f}}
\end{array}\right], \\
& \mathcal{L}_{m}:=\left[\begin{array}{ccccc}
\bar{C}_{m} & 0 & 0_{n_{z} \times 2 n_{x}} & \bar{D}_{m} & 0 \\
0 & \bar{C}_{m} & 0_{n_{z} \times 2 n_{x}} & 0 & \bar{D}_{m}
\end{array}\right], \\
& \mathcal{P}_{n}^{(s)}:=\sum_{n \in \mathcal{I}_{\mathcal{K}}^{(m)}} \pi_{m n}\left(P_{1 n}+P_{2 n}\right)+\sum_{n \in \mathcal{I}_{\mathcal{U C}}^{(m)}} \tilde{\pi}_{m n}^{(s)}\left(P_{1 n}+P_{2 n}\right)+\pi_{\mathcal{U} \mathcal{K}}^{(m s)} \underbrace{\left(P_{1 n}+P_{2 n}\right)}_{n \in \mathcal{I}_{\mathcal{U} \mathcal{K}}^{(m)}}, \\
& \pi_{\mathcal{U K}}^{(m s)}:=1-\sum_{n \in \mathcal{I}_{\mathcal{K}}^{(m)}} \pi_{m n}-\sum_{n \in \mathcal{I}_{\mathcal{U C}}^{(m)}} \tilde{\pi}_{m n}^{(s)} .
\end{aligned}
$$

Proof. Consider the following MLKF for the 2-D filtering error system in (6),

$$
V(i, j):=\sum_{k=1}^{2} V_{k}(\bar{x}(i, j+1), r(i, j+1))+\sum_{k=3}^{4} V_{k}(\bar{x}(i+1, j), r(i+1, j)),
$$

where

$$
\begin{aligned}
& V_{1}(\bar{x}(i, j+1), r(i, j+1)):=\bar{x}^{\mathrm{T}}(i, j+1) P_{1}(r(i, j+1)) \bar{x}(i, j+1), \\
& V_{2}(\bar{x}(i, j+1), r(i, j+1)):=\sum_{k=i-d_{1}}^{i-1} \bar{x}^{\mathrm{T}}(k, j+1) E^{\mathrm{T}} Q_{1} E \bar{x}(k, j+1), \\
& V_{3}(\bar{x}(i+1, j), r(i+1, j)):=\bar{x}^{\mathrm{T}}(i+1, j) P_{2}(r(i+1, j)) \bar{x}(i+1, j), \\
& V_{4}(\bar{x}(i+1, j), r(i+1, j)):=\sum_{k=j-d_{2}}^{j-1} \bar{x}^{\mathrm{T}}(i+1, k) E^{\mathrm{T}} Q_{2} E \bar{x}(i+1, k) .
\end{aligned}
$$

Then, based on the MLKF defined in (11), it is known that the 2-D filtering error system in (6) is stochastically stable with an $\mathcal{H}_{\infty}$ performance $\gamma$ under zero boundary conditions for any non-zero $w(i, j) \in l_{2}\{[0, \infty),[0, \infty)\}$ if the following inequality holds,

$$
\mathbb{J}:=\Delta V(i, j)+\|\tilde{z}\|_{\mathbb{E}_{2}}^{2}-\gamma^{2}\|\tilde{w}\|_{2}^{2}<0,
$$

where

$$
\begin{aligned}
\Delta V(i, j):= & \mathbb{E}\left\{\sum_{k=1}^{2} V_{k}(\bar{x}(i+1, j+1), r(i+1, j+1)) \mid \bar{x}(i, j+1), r(i, j+1)=m\right\} \\
& +\mathbb{E}\left\{\sum_{k=3}^{4} V_{k}(\bar{x}(i+1, j+1), r(i+1, j+1)) \mid \bar{x}(i+1, j), r(i+1, j)=m\right\} \\
& -\sum_{k=1}^{2} V_{k}(\bar{x}(i, j+1), r(i, j+1))-\sum_{k=3}^{4} V_{k}(\bar{x}(i+1, j), r(i+1, j)),
\end{aligned}
$$

and $\|\tilde{z}\|_{\mathbb{E}_{2}}$ and $\|\tilde{w}\|_{2}$ are defined in (8).

Taking the time difference of $V(i, j)$ along the trajectories of 2-D filtering error system in (6), yields

$$
\begin{aligned}
\Delta V_{1}:= & \mathbb{E}\left[V_{1}(x(i+1, j+1), r(i+1, j+1)) \mid \bar{x}(i, j+1), r(i, j+1)=m\right]-V_{1}(\bar{x}(i, j+1), r(i, j+1)) \\
= & \left.\bar{x}^{\mathrm{T}}(i+1, j+1) \sum_{n \in \mathcal{I}} \pi_{m n} P_{1 n}\right) \bar{x}(i+1, j+1)-\bar{x}^{\mathrm{T}}(i, j+1) P_{1 m} \bar{x}(i, j+1) \\
= & \left.\bar{x}^{\mathrm{T}}(i+1, j+1)\left(\sum_{n \in \mathcal{I}_{\mathcal{K}}^{(m)}} \pi_{m n} P_{1 n}+\sum_{n \in \mathcal{I}_{\mathcal{U}}^{(m)}} \sum_{s=1}^{M} \alpha_{s} \tilde{\pi}_{m n}^{(s)}\right) P_{1 n}+\sum_{n \in \mathcal{I}_{\mathcal{L}}^{(m)}} \hat{\pi}_{m n} P_{1 n}\right) \\
& \times \bar{x}(i+1, j+1)-\bar{x}^{\mathrm{T}}(i, j+1) P_{1 m} \bar{x}(i, j+1), \\
\Delta V_{2}:= & \mathbb{E}\left[V_{2}(x(i+1, j+1), r(i+1, j+1)) \mid \bar{x}(i, j+1), r(i, j+1)=m\right]-V_{2}(\bar{x}(i, j+1), r(i, j+1)) \\
= & \bar{x}^{\mathrm{T}}(i, j+1) E^{\mathrm{T}} Q_{1} E \bar{x}(i, j+1)-\bar{x}^{\mathrm{T}}\left(i-d_{1}, j+1\right) E^{\mathrm{T}} Q_{1} E \bar{x}\left(i-d_{1}, j+1\right),
\end{aligned}
$$




$$
\begin{aligned}
\Delta V_{3}:= & \mathbb{E}\left[V_{3}(x(i+1, j+1), r(i+1, j+1)) \mid \bar{x}(i+1, j), r(i+1, j)=m\right]-V_{3}(\bar{x}(i+1, j), r(i+1, j)) \\
= & \left.\bar{x}^{\mathrm{T}}(i+1, j+1) \sum_{n \in \mathcal{I}} \pi_{m n} P_{2 n}\right) \bar{x}(i+1, j+1)-\bar{x}^{\mathrm{T}}(i+1, j) P_{2 m} \bar{x}(i+1, j) \\
= & \left.\bar{x}^{\mathrm{T}}(i+1, j+1)\left(\sum_{n \in \mathcal{I}_{\kappa}^{(m)}} \pi_{m n} P_{2 n}+\sum_{n \in \mathcal{I}_{\mathcal{L}}^{(m)}} \sum_{s=1}^{M} \alpha_{s} \tilde{\pi}_{m n}^{(s)}\right) P_{2 n}+\sum_{n \in \mathcal{I}_{\mathcal{L}}^{(m)}} \hat{\pi}_{m n} P_{2 n}\right) \\
& \times \bar{x}(i+1, j+1)-\bar{x}^{\mathrm{T}}(i+1, j) P_{2 m} \bar{x}(i+1, j), \\
\Delta V_{4}:= & \mathbb{E}\left[V_{4}(x(i+1, j+1), r(i+1, j+1)) \mid \bar{x}(i+1, j), r(i+1, j)=m\right]-V_{4}(\bar{x}(i+1, j), r(i+1, j)) \\
= & \bar{x}^{\mathrm{T}}(i+1, j) E^{\mathrm{T}} Q_{2} E \bar{x}(i+1, j)-\bar{x}^{\mathrm{T}}\left(i+1, j-d_{2}\right) E^{\mathrm{T}} Q_{2} E \bar{x}\left(i+1, j-d_{2}\right) .
\end{aligned}
$$

Therefore, based on the MLKF defined in (11), together with consideration of (6) and (14)-(17), we have,

$$
\sqrt{ }=\varsigma^{\mathrm{T}}(i, j)\left[\mathcal{A}_{m}^{\mathrm{T}}\left(\overline{\mathcal{P}}_{1 n}+\overline{\mathcal{P}}_{2 n}\right) \mathcal{A}_{m}+\mathcal{L}_{m}^{\mathrm{T}} \mathcal{L}_{m}+\Theta_{m}\right] \varsigma(i, j), \quad m, n \in \mathcal{I},
$$

where

$$
\begin{aligned}
& \varsigma(i, j):=\left[\begin{array}{lllll}
\bar{x}^{\mathrm{T}}(i, j+1) & \bar{x}^{\mathrm{T}}(i+1, j) & x^{\mathrm{T}}\left(i-d_{1}, j+1\right) x^{\mathrm{T}}\left(i+1, j-d_{2}\right) & w^{\mathrm{T}}(i, j+1) & w^{\mathrm{T}}(i+1, j)
\end{array}\right]^{\mathrm{T}}, \\
& \Theta_{m}:=\operatorname{diag}\left\{-P_{1 m}+E^{\mathrm{T}} Q_{1} E,-P_{2 m}+E^{\mathrm{T}} Q_{2} E,-Q_{1},-Q_{2},-\gamma^{2} I,-\gamma^{2} I\right\}, \\
& \mathcal{A}_{m}:=\left[\begin{array}{llllll}
\bar{A}_{1 m} & \bar{A}_{2 m} & \bar{A}_{d 1 m} & \bar{A}_{d 2 m} & \bar{B}_{1 m} & \bar{B}_{1 m}
\end{array}\right] \text {, } \\
& \mathcal{L}_{m}:=\left[\begin{array}{ccccc}
\bar{C}_{m} & 0 & 0_{n_{2} \times 2 n_{x}} & \bar{D}_{m} & 0 \\
0 & \bar{C}_{m} & 0_{n_{2} \times 2 n_{x}} & 0 & \bar{D}_{m}
\end{array}\right], \\
& \left.\overline{\mathcal{P}}_{l n}:=\sum_{n \in \mathcal{I}_{\kappa}^{(m)}} \pi_{m n} P_{l n}+\sum_{n \in \mathcal{I}_{\mathcal{L C}}^{(m)}} \sum_{s=1}^{M} \alpha_{s} \tilde{\pi}_{m n}^{(s)}\right) P_{l n}+\sum_{n \in \mathcal{I}_{u k}^{(m)}} \hat{\pi}_{m n} P_{l n}, \quad l=1,2 .
\end{aligned}
$$

Considering the fact that $0 \leqslant \alpha_{s} \leqslant 1, \sum_{s=1}^{M} \alpha_{s}=1$, and $0 \leqslant \frac{\hat{\pi}_{m m}}{\pi_{u \mathcal{L}}^{(m)}} \leqslant 1, \sum_{n \in \mathcal{I}_{\mathcal{L}}^{(m)}} \frac{\hat{\pi}_{m n}}{\pi_{u \mathcal{k}}^{(m)}}=1$, (18) can be rewritten as,

$$
\mathbb{J}=\sum_{s=1}^{M} \alpha_{s} \sum_{n \in \mathcal{I}_{\mathcal{L}}^{(m)}} \frac{\hat{\pi}_{m n}}{\pi_{\mathcal{U K}}^{(m)}}\left[\varsigma^{\mathrm{T}}(i, j)\left[\mathcal{A}_{m}^{\mathrm{T}} \mathcal{P}_{n}^{(s)} \mathcal{A}_{m}+\mathcal{L}_{m}^{\mathrm{T}} \mathcal{L}_{m}+\Theta_{m}\right] \varsigma(i, j)\right], \quad m \in \mathcal{I}, \quad n \in \mathcal{I}_{\mathcal{U K}}^{(m)}, \quad s=1, \ldots, M,
$$

where

$$
\begin{aligned}
\mathcal{P}_{n}^{(s)} & :=\sum_{n \in \mathcal{I}_{\kappa}^{(m)}} \pi_{m n}\left(P_{1 n}+P_{2 n}\right)+\sum_{n \in \mathcal{I}_{\mathcal{U}}^{(m)}} \tilde{\pi}_{m n}^{(s)}\left(P_{1 n}+P_{2 n}\right)+\pi_{\mathcal{U}}^{(m s)} \underbrace{\left(P_{1 n}+P_{2 n}\right.}_{n \in \mathcal{I}_{\mathcal{L}}^{(m)}}), \\
\pi_{\mathcal{U}}^{(m s)} & :=1-\sum_{n \in \mathcal{I}_{\kappa}^{(m)}} \pi_{m n}-\sum_{n \in \mathcal{I}_{\mathcal{L}}^{(m)}} \tilde{\pi}_{m n}^{(s)} .
\end{aligned}
$$

According to (20), it is easy to see that (13) holds if and only if $\forall s=1, \ldots, M$,

$$
\varsigma^{\mathrm{T}}(i, j)\left[\mathcal{A}_{m}^{\mathrm{T}} \mathcal{P}_{n}^{(s)} \mathcal{A}_{m}+\mathcal{L}_{m}^{\mathrm{T}} \mathcal{L}_{m}+\Theta_{m}\right] \varsigma(i, j)<0, \quad m \in \mathcal{I}, \quad n \in \mathcal{I}_{\mathcal{U}}^{(m)},
$$

which is implied by (9). This completes the proof.

Remark 2. By fully exploiting the properties of TPM, together with the convexification of uncertain domains, a new sufficient condition for $\mathcal{H}_{\infty}$ performance analysis has been derived for the 2-D filtering error system $(\bar{\Sigma})$ with state-delays and deficient mode information in Proposition 1. It is noted that there exist product terms between the Lyapunov matrices and system matrices in condition (9), which brings some difficulties for filter synthesis problem. In the following, by some matrix inequality linearisation techniques, two approaches for the filter design will be proposed.

Before proceeding further, we present the following Projection lemma, which will be used in the filter synthesis procedure.

Lemma 1 ([3,21]). Given matrices $\mathbb{W}=\mathbb{W}^{\mathrm{T}} \in R^{n \times n}, \mathbb{U} \in R^{k \times n}, \mathbb{V} \in R^{m \times n}$, the following linear matrix inequality problem,

$$
\mathbb{W}+\mathbb{U}^{\mathrm{T}} \mathbb{X}^{\mathrm{T}} \mathbb{V}+\mathbb{V}^{\mathrm{T}} \mathbb{X} \mathbb{U}<0
$$

is solvable with respect to the variable $\mathbb{X}$ if and only if 


$$
\begin{aligned}
& \mathbb{U}_{\perp}^{\mathrm{T}} \mathbb{W}_{\perp}<0 \quad \text { if } \mathbb{V}_{\perp}=0, \quad \mathbb{U}_{\perp} \neq 0, \\
& \mathbb{V}_{\perp}^{\mathrm{T}} \mathbb{W V}_{\perp}<0 \quad \text { if } \mathbb{U}_{\perp}=0, \quad \mathbb{V}_{\perp} \neq 0, \\
& \mathbb{U}_{\perp}^{\mathrm{T}} \mathbb{W U}_{\perp}<0, \quad \mathbb{V}_{\perp}^{\mathrm{T}} \mathbb{W V}_{\perp}<0 \quad \text { if } \mathbb{V}_{\perp} \neq 0, \quad \mathbb{U}_{\perp} \neq 0,
\end{aligned}
$$

where $\mathbb{U}_{\perp}$ and $\mathbb{V}_{\perp}$ denote the right null spaces of $\mathbb{U}$ and $\mathbb{V}$, respectively.

\section{2. $\mathcal{H}_{\infty}$ filter synthesis}

In this subsection, based on the Projection lemma and some bounding inequalities, two approaches for the solvability of the $\mathcal{H}_{\infty}$ filtering problem will be proposed. It will be shown that the parameterised representations of the filter gains can be constructed in terms of the feasible solutions to a set of strict linear matrix inequalities (LMIs).

Theorem 1. Consider the 2-D MJLS in (1) with state-delays and deficient mode information, and filter in the form of (5). The filtering error system in (6) is stochastically stable with an $\mathcal{H}_{\infty}$ performance $\gamma$, if there exist positive-definite symmetric matrices $\left\{P_{1 m}, P_{2 m}\right\} \in R^{\left(n_{x}+n_{f}\right) \times\left(n_{x}+n_{f}\right)},\left\{Q_{1}, Q_{2}\right\} \in R^{n_{x} \times n_{x}}$, and matrices $G_{m(1)} \in R^{n_{x} \times n_{x}}, G_{m(2)} \in R^{n_{f} \times n_{f}}, G_{m(3)} \in R^{n_{f} \times n_{x}}, U_{m} \in R^{\left(4 n_{x}+2 n_{f}+2 n_{w}\right) \times n_{x}}$, $\left\{\bar{A}_{f 1 m}, \bar{A}_{f 2 m}\right\} \in R^{n_{f} \times n_{f}},\left\{\bar{B}_{f 1 m}, \bar{B}_{f 2 m}\right\} \in R^{n_{f} \times n_{y}}, C_{f m} \in R^{n_{z} \times n_{f}}$, and $D_{f m} \in R^{n_{z} \times n_{y}}, m \in \mathcal{I}$, such that the following LMIs hold,

$$
\left[\begin{array}{cc}
-I & \overline{\mathcal{L}}_{m} \\
* & \bar{\Theta}_{m n}^{(s)}+\operatorname{Sym}\left\{\overline{\mathcal{A}}_{m}\right\}
\end{array}\right]<0, \quad m \in \mathcal{I}, \quad n \in \mathcal{I}_{\mathcal{U}}^{(m)}, \quad s=1, \ldots, M,
$$

where

$$
\begin{aligned}
& \bar{\Theta}_{m n}^{(s)}:=\operatorname{diag}\left\{\mathcal{P}_{n}^{(s)},-P_{1 m}+E^{\mathrm{T}} Q_{1} E,-P_{2 m}+E^{\mathrm{T}} Q_{2} E,-Q_{1},-Q_{2},-\gamma^{2} I,-\gamma^{2} I\right\}, \\
& \overline{\mathcal{A}}_{m}:=\left[\begin{array}{lllllll}
\overline{\mathcal{G}}_{m} & \overline{\mathcal{A}}_{1 m} & \overline{\mathcal{A}}_{2 m} & \overline{\mathcal{A}}_{d 1 m} & \overline{\mathcal{A}}_{d 2 m} & \overline{\mathcal{B}}_{1 m} & \overline{\mathcal{B}}_{2 m}
\end{array}\right], \\
& \overline{\mathcal{L}}_{m}:=\left[\begin{array}{cccccc}
0_{n_{z} \times\left(n_{x}+n_{f}\right)} & \bar{C}_{m} & 0 & 0_{n_{z} \times 2 n_{x}} & \bar{D}_{m} & 0 \\
0_{n_{z} \times\left(n_{x}+n_{f}\right)} & 0 & \bar{C}_{m} & 0_{n_{z} \times 2 n_{x}} & 0 & \bar{D}_{m}
\end{array}\right], \\
& \overline{\mathcal{G}}_{m}:=\left[\begin{array}{cc}
-G_{m(1)} & -H G_{m(2)} \\
-G_{m(3)} & -G_{m(2)} \\
-U_{m} & 0
\end{array}\right], \quad H:=\left[\begin{array}{c}
I_{n_{f}} \\
0_{\left(n_{x}-n_{f}\right) \times n_{f}}
\end{array}\right], \\
& \overline{\mathcal{A}}_{1 m}:=\left[\begin{array}{cc}
G_{m(1)} A_{1 m}+H \bar{B}_{f 1 m} C_{m} & H \bar{A}_{f 1 m} \\
G_{m(3)} A_{1 m}+\bar{B}_{f 1 m} C_{m} & \bar{A}_{f 1 m} \\
U_{m} A_{1 m} & 0
\end{array}\right] \text {, } \\
& \overline{\mathcal{A}}_{2 m}:=\left[\begin{array}{cc}
G_{m(1)} A_{2 m}+H \bar{B}_{f 2 m} C_{m} & H \bar{A}_{f 2 m} \\
G_{m(3)} A_{2 m}+\bar{B}_{f 2 m} C_{m} & \bar{A}_{f 2 m} \\
U_{m} A_{2 m} & 0
\end{array}\right] \text {, } \\
& \overline{\mathcal{A}}_{d 1 m}:=\left[\begin{array}{c}
G_{m(1)} A_{d 1 m} \\
G_{m(3)} A_{d 1 m} \\
U_{m} A_{d 1 m}
\end{array}\right], \quad \overline{\mathcal{A}}_{d 2 m}:=\left[\begin{array}{c}
G_{m(1)} A_{d 2 m} \\
G_{m(3)} A_{d 2 m} \\
U_{m} A_{d 2 m}
\end{array}\right], \\
& \overline{\mathcal{B}}_{1 m}:=\left[\begin{array}{c}
G_{m(1)} B_{1 m}+H \bar{B}_{f 1 m} D_{m} \\
G_{m(3)} B_{1 m}+\bar{B}_{f 1 m} D_{m} \\
U_{m} B_{1 m}
\end{array}\right], \quad \overline{\mathcal{B}}_{2 m}:=\left[\begin{array}{c}
G_{m(1)} B_{2 m}+H \bar{B}_{f 2 m} D_{m} \\
G_{m(3)} B_{2 m}+\bar{B}_{f 2 m} D_{m} \\
U_{m} B_{2 m}
\end{array}\right], \\
& \mathcal{P}_{n}^{(s)}:=\sum_{n \in \mathcal{I}_{\mathcal{K}}^{(m)}} \pi_{m n}\left(P_{1 n}+P_{2 n}\right)+\sum_{n \in \mathcal{I}_{\mathcal{U C}}^{(m)}} \tilde{\pi}_{m n}^{(s)}\left(P_{1 n}+P_{2 n}\right)+\pi_{\mathcal{U K}}^{(m s)} \underbrace{\left(P_{1 n}+P_{2 n}\right)}_{n \in \mathcal{I}_{\mathcal{L}}^{(m)}},
\end{aligned}
$$

with $E, \bar{C}_{m}, \bar{D}_{m}$, and $\pi_{\mathcal{U}}^{(m)}$ defined in (7) and (10), respectively. Moreover, if the above conditions have a set of feasible solutions $\left(P_{1 m}, P_{2 m}, Q_{1}, Q_{2}, G_{m(1)}, G_{m(2)}, G_{m(3)}, U_{m}, \bar{A}_{f 1 m}, \bar{A}_{f 2 m}, \bar{B}_{f 1 m}, \bar{B}_{f 2 m}, C_{f m}, D_{f m}\right)$, then an admissible $n_{f}$-order filter in the form of (5) can be constructed as,

$$
A_{f 1 m}=G_{m(2)}^{-1} \bar{A}_{f 1 m}, A_{f 2 m}=G_{m(2)}^{-1} \bar{A}_{f 2 m}, B_{f 1 m}=G_{m(2)}^{-1} \bar{B}_{f 1 m}, B_{f 2 m}=G_{m(2)}^{-1} \bar{B}_{f 2 m},
$$

and $C_{f m}$ and $D_{f m}$ can be obtained directly from (23). 
Proof. It follows from Proposition 1 that if we can show (9), then the claimed results follow. Nevertheless, it is easy to see that in (9), the system matrices are coupled with Lyapunov matrices. Therefore, it is anticipated that the conditions in (9) are not convenient when using for filter design purpose. To resolve this problem, in the following, the Projection lemma (Lemma 1) will be utilised to eliminate the coupling between the system matrices and Lyapunov matrices. To this end, we reformulate the inequality in (9) as,

$$
\left[\begin{array}{c}
\mathcal{A}_{m} \\
I_{\left(4 n_{x}+2 n_{f}+2 n_{w}\right)}
\end{array}\right]^{\mathrm{T}}\left[\begin{array}{cc}
\mathcal{P}_{n}^{(s)} & 0 \\
* & \mathcal{L}_{m}^{\mathrm{T}} \mathcal{L}_{m}+\Theta_{m}
\end{array}\right]\left[\begin{array}{c}
\mathcal{A}_{m} \\
I_{\left(4 n_{x}+2 n_{f}+2 n_{w}\right)}
\end{array}\right]<0, \quad m \in \mathcal{I}, \quad n \in \mathcal{I}_{\mathcal{U}}^{(m)}, \quad s=1, \ldots, M,
$$

where $\mathcal{A}_{m}, \mathcal{L}_{m}, \mathcal{P}_{n}^{(s)}$ and $\Theta_{m}$ are defined in (10).

Explicit null space calculation yields,

$$
\left[\begin{array}{ll}
-I\left(n_{x}+n_{f}\right) & \mathcal{A}_{m}
\end{array}\right]_{\perp}=\left[\begin{array}{c}
\mathcal{A}_{m} \\
I_{\left(4 n_{x}+2 n_{f}+2 n_{w}\right)}
\end{array}\right] .
$$

Then, assigning

$$
\begin{aligned}
& \mathbb{W}\left[\begin{array}{cc}
\mathcal{P}_{n}^{(s)} & 0 \\
* & \mathcal{L}_{m}^{\mathrm{T}} \mathcal{L}_{m}+\Theta_{m}
\end{array}\right], \\
& \mathbb{X} \leftarrow G_{m} \in R^{\left(5 n_{x}+3 n_{f}+2 n_{w}\right) \times\left(n_{x}+n_{f}\right)}, \\
& \mathbb{U} \quad\left[\begin{array}{ll}
-I_{\left(n_{x}+n_{f}\right)} & \mathcal{A}_{m}
\end{array}\right], \\
& \mathbb{U}_{\perp}\left[\begin{array}{c}
\mathcal{A}_{m} \\
I_{\left(4 n_{x}+2 n_{f}+2 n_{w}\right)}
\end{array}\right], \\
& \text { V I, } \\
& \mathbb{V}_{\perp} \quad 0
\end{aligned}
$$

and applying Projection lemma to (26), yield

$$
\left[\begin{array}{cc}
\mathcal{P}_{n}^{(s)} & 0 \\
* & \mathcal{L}_{m}^{\mathrm{T}} \mathcal{L}_{m}+\Theta_{m}
\end{array}\right]+\operatorname{Sym}\left\{G_{m}\left[-I_{\left(n_{x}+n_{f}\right)} \quad \mathcal{A}_{m}\right]\right\}<0, \quad m \in \mathcal{I}, \quad n \in \mathcal{I}_{\mathcal{U} \mathcal{K}}^{(m)}, \quad s=1, \ldots, M .
$$

Now, it is easy to see that in (27), the Lyapunov matrices have been separated from the system matrices. Thus, the main task hereafter is to convexify the products of the system matrices with the slack variables.

For simplicity in the filter design procedure, we first specify the slack variables as,

$$
G_{m}=\left[\begin{array}{c}
{\left[\begin{array}{cc}
G_{m(1)} & H G_{(2)} \\
G_{m(3)} & G_{(4)}
\end{array}\right]} \\
0_{\left(4 n_{x}+2 n_{f}+2 n_{w}\right) \times\left(n_{x}+n_{f}\right)}
\end{array}\right], \quad m \in \mathcal{I},
$$

where $H:=\left[\begin{array}{ll}I_{n_{f}} & 0_{n_{f} \times\left(n_{x}-n_{f}\right.}\end{array}\right]^{\mathrm{T}}, G_{m(1)} \in R^{n_{x} \times n_{x}}, G_{(2)} \in R^{n_{f} \times n_{f}}, G_{m(3)} \in R^{n_{f} \times n_{x}}$, and $G_{(4)} \in R^{n_{f} \times n_{f}}$. Then, for matrix inequality linearisation purpose, similar to $[19,20,33]$, performing a congruent transformation to

$$
\left[\begin{array}{cc}
G_{m(1)}+G_{m(1)}^{\mathrm{T}} & H G_{(2)}+G_{m(3)}^{\mathrm{T}} \\
* & G_{(4)}+G_{(4)}^{\mathrm{T}}
\end{array}\right]
$$

by $\operatorname{diag}\left\{I_{n_{x}}, G_{(2)} G_{(4)}^{-1}\right\}$ yields

$$
\left[\begin{array}{cc}
G_{m(1)}+G_{m(1)}^{\mathrm{T}} & H G_{(2)} G_{(4)}^{-\mathrm{T}} G_{(2)}^{\mathrm{T}}+G_{m(3)}^{\mathrm{T}} G_{(4)}^{-\mathrm{T}} G_{(2)}^{\mathrm{T}} \\
* & G_{(2)} G_{(4)}^{-\mathrm{T}} G_{(2)}^{\mathrm{T}}+G_{(2)} G_{(4)}^{-1} G_{(2)}^{\mathrm{T}}
\end{array}\right]:=\left[\begin{array}{cc}
G_{m(1)}+G_{m(1)}^{\mathrm{T}} & H{\overline{G_{(2)}}}+\bar{G}_{m(3)}^{\mathrm{T}} \\
* & \underline{\underline{\overline{G_{(2)}}}+\bar{G}_{(2)}^{\mathrm{T}}}
\end{array}\right] .
$$

Thus, instead of (28), one can directly specify the matrix $G_{m}$ of the following form without loss of generality,

$$
G_{m}=\left[\begin{array}{cc}
{\left[\begin{array}{cc}
G_{m(1)} & H G_{m(2)} \\
G_{m(3)} & G_{m(2)}
\end{array}\right]} \\
0_{\left(4 n_{x}+2 n_{f}+2 n_{w}\right) \times\left(n_{x}+n_{f}\right)}
\end{array}\right], \quad m \in \mathcal{I} .
$$

It is noted that in this way the matrix variable $G_{m(2)}$ can be absorbed by the filter gain variables $A_{f 1 m}, A_{f 2 m}, B_{f 1 m}$ and $B_{f 2 m}$ by introducing

$$
\bar{A}_{f 1 m}:=G_{m(2)} A_{f 1 m}, \quad \bar{A}_{f 2 m}:=G_{m(2)} A_{f 2 m}, \quad \bar{B}_{f 1 m}:=G_{m(2)} B_{f 1 m}, \quad \bar{B}_{f 2 m}:=G_{m(2)} B_{f 2 m} .
$$


This feature enables one to make no congruent transformation to the original matrix inequality and all the slack variables can be set as Markovian switching.

Moreover, it is noted that the filter gains are not involved in the first row of the system matrices $\bar{A}_{1 m}, \bar{A}_{2 m}, \bar{A}_{d 1 m}, \bar{A}_{d 2 m}, \bar{B}_{1 m}$ and $\bar{B}_{2 m}$ in (7), such that to further reduce the design conservatism, we can specify the slack variable as

$$
G_{m}=\left[\begin{array}{cc}
G_{m(1)} & H G_{m(2)} \\
G_{m(3)} & G_{m(2)} \\
U_{m} & 0
\end{array}\right], \quad m \in \mathcal{I} .
$$

Then, by substituting the matrix $G_{m}$ defined in (33) into (27) and applying Schur complement, together with consideration of (32), one readily obtains (23).

On the other hand, the conditions in (23) imply that $-G_{m(2)}-G_{m(2)}^{\mathrm{T}}<0$, which means that $G_{m(2)}$ is nonsingular. Then, the filter gains can be constructed by (25). The proof is thus completed.

Remark 3. Theorem 1 provides a sufficient condition for the solvability of $n_{f}$-order $\mathcal{H}_{\infty}$ filtering for the 2-D MJLS in (1) with state-delays and deficient mode information. It is noted that the $\mathcal{H}_{\infty}$ filtering problem for 2-D discrete-time MJLSs has been considered in [33]. However, there are some remarkable differences between our results and those in [33]. Firstly, the statedelays are considered in system (1), whereas the 2-D delay-free MJLSs were considered in [33]. Secondly, in this paper the exactly known, partially unknown and uncertain transition probabilities (TPs) have been simultaneously incorporated into the TPM for 2-D MJLSs, which is more general and practical for engineering applications, while the TPs considered in [33] are assumed to be completely known. Moreover, based on Proposition 1 combined with Projection lemma, Theorem 1 gives a unified formulation of full-order and reduced-order $\mathcal{H}_{\infty}$ filtering design for 2-D MJLSs with state-delays and deficient mode information, while in [33], only the full-order $\mathcal{H}_{\infty}$ filtering design was investigated by a traditional decoupling inequality. It will be shown in the simulation section that the above points are crucial to reduce the conservatism of filtering design for the underlying 2-D MJLSs.

It is noted that the conditions given in Theorem 1 are derived based on the Projection lemma. For comparison purposes, in the following, we also propose another $n_{f}$-order $\mathcal{H}_{\infty}$ filtering design method based on the same performance criterion presented in Proposition 1 and a traditional decoupling inequality, but without using Projection lemma. The corresponding result is summarised in the following theorem.

Theorem 2. Consider the 2-D MJLS in (1) with state-delays and deficient mode information, and filter in the form of (5). The filtering error system in (6) is stochastically stable with an $\mathcal{H}_{\infty}$ performance $\gamma$, if there exist positive-definite symmetric matrices $\left\{P_{1 m}, P_{2 m}\right\} \in R^{\left(n_{x}+n_{f}\right) \times\left(n_{x}+n_{f}\right)},\left\{Q_{1}, Q_{2}\right\} \in R^{n_{x} \times n_{x}}$, and matrices $G_{m(1)} \in R^{n_{x} \times n_{x}}, G_{m(2)} \in R^{n_{f} \times n_{f}}, G_{m(3)} \in R^{n_{f} \times n_{x}},\left\{\bar{A}_{f 1 m}, \bar{A}_{f 2 m}\right\} \in R^{n_{f} \times n_{f}}$, $\left\{\bar{B}_{f 1 m}, \bar{B}_{f 2 m}\right\} \in R^{n_{f} \times n_{y}}, C_{f m} \in R^{n_{z} \times n_{f}}$, and $D_{f m} \in R^{n_{z} \times n_{y}}, m \in \mathcal{I}$, such that the following LMIs hold,

$$
\left[\begin{array}{ccc}
-I & 0 & \mathcal{L}_{m} \\
* & \mathcal{P}_{n}^{(s)}-\operatorname{Sym}\left\{G_{m}\right\} & \hat{\mathcal{A}}_{m} \\
* & * & \Theta_{m}
\end{array}\right]<0, \quad m \in \mathcal{I}, \quad n \in \mathcal{I}_{\mathcal{U}}^{(m)}, \quad s=1, \ldots, M
$$

where

$$
\begin{aligned}
& \Theta_{m}:=\operatorname{diag}\left\{-P_{1 m}+E^{\mathrm{T}} Q_{1} E,-P_{2 m}+E^{\mathrm{T}} Q_{2} E,-Q_{1},-Q_{2},-\gamma^{2} I,-\gamma^{2} I\right\}, \\
& \hat{\mathcal{A}}_{m}:=\left[\begin{array}{llllll}
\hat{\mathcal{A}}_{1 m} & \widehat{\mathcal{A}}_{2 m} & \widehat{\mathcal{A}}_{d 1 m} & \widehat{\mathcal{A}}_{d 2 m} & \widehat{\mathcal{B}}_{1 m} & \widehat{\mathcal{B}}_{2 m}
\end{array}\right] \text {, } \\
& \mathcal{L}_{m}:=\left[\begin{array}{ccccc}
\bar{C}_{m} & 0 & 0_{n_{z} \times 2 n_{x}} & \bar{D}_{m} & 0 \\
0 & \bar{C}_{m} & 0_{n_{z} \times 2 n_{x}} & 0 & \bar{D}_{m}
\end{array}\right], \\
& G_{m}:=\left[\begin{array}{cc}
-G_{m(1)} & -H G_{m(2)} \\
-G_{m(3)} & -G_{m(2)}
\end{array}\right], \quad H:=\left[\begin{array}{c}
I_{n_{f}} \\
0_{\left(n_{x}-n_{f}\right) \times n_{f}}
\end{array}\right], \\
& \widehat{\mathcal{A}}_{1 m}:=\left[\begin{array}{cc}
G_{m(1)} A_{1 m}+H \bar{B}_{f 1 m} C_{m} & H \bar{A}_{f 1 m} \\
G_{m(3)} A_{1 m}+\bar{B}_{f 1 m} C_{m} & \bar{A}_{f 1 m}
\end{array}\right] \text {, } \\
& \widehat{\mathcal{A}}_{2 m}:=\left[\begin{array}{cc}
G_{m(1)} A_{2 m}+H \bar{B}_{f 2 m} C_{m} & H \bar{A}_{f 2 m} \\
G_{m(3)} A_{2 m}+\bar{B}_{f 2 m} C_{m} & \bar{A}_{f 2 m}
\end{array}\right], \\
& \widehat{\mathcal{A}}_{d 1 m}:=\left[\begin{array}{l}
G_{m(1)} A_{d 1 m} \\
G_{m(3)} A_{d 1 m}
\end{array}\right], \quad \widehat{\mathcal{A}}_{d 2 m}:=\left[\begin{array}{l}
G_{m(1)} A_{d 2 m} \\
G_{m(3)} A_{d 2 m}
\end{array}\right], \\
& \widehat{\mathcal{B}}_{1 m}:=\left[\begin{array}{c}
G_{m(1)} B_{1 m}+H \bar{B}_{f 1 m} D_{m} \\
G_{m(3)} B_{1 m}+\bar{B}_{f 1 m} D_{m}
\end{array}\right], \quad \widehat{\mathcal{B}}_{2 m}:=\left[\begin{array}{c}
G_{m(1)} B_{2 m}+H \bar{B}_{f 2 m} D_{m} \\
G_{m(3)} B_{2 m}+\bar{B}_{f 2 m} D_{m}
\end{array}\right], \\
& \mathcal{P}_{n}^{(s)}:=\sum_{n \in \mathcal{I}_{\mathcal{K}}^{(m)}} \pi_{m n}\left(P_{1 n}+P_{2 n}\right)+\sum_{n \in \mathcal{I}_{\mathcal{H C}}^{(m)}} \tilde{\pi}_{m n}^{(s)}\left(P_{1 n}+P_{2 n}\right)+\pi_{\mathcal{L}}^{(m s)} \underbrace{\left(P_{1 n}+P_{2 n}\right)}_{n \in \mathcal{I}_{u k}^{(m)}},
\end{aligned}
$$


with $E, \bar{C}_{m}, \bar{D}_{m}$, and $\pi_{\mathcal{U}}^{(m s)}$ defined in (7) and (10), respectively. Moreover, if the above conditions have a set of feasible solutions $\left(P_{1 m}, P_{2 m}, Q_{1}, Q_{2}, G_{m(1)}, G_{m(2)}, G_{m(3)}, \bar{A}_{f 1 m}, \bar{A}_{f 2 m}, \bar{B}_{f 1 m}, \bar{B}_{f 2 m}, C_{f m}, D_{f m}\right)$, then an admissible $n_{f}$-order filter in the form of (5) can be constructed as,

$$
A_{f 1 m}=G_{m(2)}^{-1} \bar{A}_{f 1 m}, \quad A_{f 2 m}=G_{m(2)}^{-1} \bar{A}_{f 2 m}, \quad B_{f 1 m}=G_{m(2)}^{-1} \bar{B}_{f 1 m}, \quad B_{f 2 m}=G_{m(2)}^{-1} \bar{B}_{f 2 m}
$$

and $C_{f m}$ and $D_{f m}$ can be obtained directly from (34).

Proof. By Schur complement, (9) is equivalent to

$$
\left[\begin{array}{ccc}
-I & 0 & \mathcal{L}_{m} \\
* & -\left(\mathcal{P}_{n}^{(s)}\right)^{-1} & \mathcal{A}_{m} \\
* & * & \Theta_{m}
\end{array}\right]<0, \quad m \in \mathcal{I}, \quad n \in \mathcal{I}_{\mathcal{U} \mathcal{K}}^{(m)}, \quad s=1, \ldots, M,
$$

where $\mathcal{A}_{m}, \mathcal{L}_{m}, \mathcal{P}_{n}^{(s)}$ and $\Theta_{m}$ are defined in (10).

For matrix inequality linearisation purpose, performing a congruent transformation to (37) by $\operatorname{diag}\left\{I_{2 n_{z}}, G_{m}, I\left(4 n_{x}+2 n_{f}+2 n_{w}\right)\right\}$, and it follows from

$$
\left(\mathcal{P}_{n}^{(s)}-G_{m}\right)^{\mathrm{T}}\left(\mathcal{P}_{n}^{(s)}\right)^{-1}\left(\mathcal{P}_{n}^{(s)}-G_{m}\right) \geqslant 0
$$

that

$$
-G_{m}^{\mathrm{T}}\left(\mathcal{P}_{n}^{(s)}\right)^{-1} G_{m} \leqslant \mathcal{P}_{n}^{(s)}-G_{m}-G_{m}^{\mathrm{T}}
$$

Based on (39), we have that the following inequality implies (37),

$$
\left[\begin{array}{ccc}
-I & 0 & \mathcal{L}_{m} \\
* & \mathcal{P}_{n}^{(s)}-\operatorname{Sym}\left\{G_{m}\right\} & G_{m} \mathcal{A}_{m} \\
* & * & \Theta_{m}
\end{array}\right]<0, \quad m \in \mathcal{I}, \quad n \in \mathcal{I}_{\mathcal{U K}}^{(m)}, \quad s=1, \ldots, M .
$$

Then, similar to the manipulations given in the proof of Theorem 1, we can specify the slack variable $G_{m}$ as follows without loss of generality,

$$
G_{m}:=\left[\begin{array}{cc}
G_{m(1)} & H G_{m(2)} \\
G_{m(3)} & G_{m(2)}
\end{array}\right]
$$

where $H:=\left[\begin{array}{ll}I_{n_{f}} & 0_{n_{f} \times\left(n_{x}-n_{f}\right)}\end{array}\right]^{\mathrm{T}}$.

Now, substituting the slack variable $G_{m}$ into (40) and defining the following matrices

$$
\bar{A}_{f 1 m}:=G_{m(2)} A_{f 1 m}, \quad \bar{A}_{f 2 m}:=G_{m(2)} A_{f 2 m}, \quad \bar{B}_{f 1 m}:=G_{m(2)} B_{f 1 m}, \quad \bar{B}_{f 2 m}:=G_{m(2)} B_{f 2 m},
$$

one can readily obtain (34). In addition, since $G_{m(2)}$ are nonsingular as implied by (34), it is easy to see that the filter gains can be constructed by (36). The proof is thus completed.

Remark 4. Based on a traditional decoupling inequality (39), Theorem 2 provides another sufficient condition for the solvability of $n_{f}$-order $\mathcal{H}_{\infty}$ filtering of 2-D MJLS (1) with state-delays and deficient mode information. Nevertheless, by observing the conditions given in Theorems 1 and 2 carefully, it is interesting to see that Theorem 2 is actually a special case of Theorem 1 with $U_{m}=0$. It will be shown in the simulation section that Theorem 1 is generally less conservative than Theorem 2 due to the more freedom in the solution space.

\section{Simulation studies}

In this section, we demonstrate the effectiveness of the proposed approaches in this paper via a simulation example.

Consider a two-dimensional (2-D) Markovian jump linear system (MJLS) with state-delays in the form of (1) with parameters as follows, 


$$
\begin{aligned}
& {\left[\begin{array}{ccc}
A_{11} & A_{d 11} & B_{11} \\
A_{21} & A_{d 21} & B_{21} \\
L_{1} & C_{1} & D_{1}
\end{array}\right]=\left[\begin{array}{ccccc}
0.3 & 0 & 0.15 & 0 & 0.3 \\
0.2 & 0.1 & 0 & 0.1 & 0.5 \\
0.1 & 0 & 0 & 0.1 & 0.2 \\
0.2 & 0.2 & 0 & 0.1 & 0.4 \\
-1 & 1 & 1 & 0 & 0 \\
0 & -0.8 & 1 & 0.6 & 0.3
\end{array}\right],} \\
& {\left[\begin{array}{ccc}
A_{12} & A_{d 12} & B_{12} \\
A_{22} & A_{d 22} & B_{22} \\
L_{2} & C_{2} & D_{2}
\end{array}\right]=\left[\begin{array}{ccccc}
0.2 & 0 & 0.1 & 0 & 0.2 \\
0.1 & 0.3 & 0 & 0.1 & 0.5 \\
0.2 & 0 & 0 & 0 & 0.5 \\
0.1 & 0.1 & 0 & 0.1 & 0.1 \\
-1.5 & 0.3 & 0.2 & 0.5 & 0 \\
0 & -0.5 & 0.5 & 0.6 & 0.5
\end{array}\right],} \\
& {\left[\begin{array}{ccc}
A_{13} & A_{d 13} & B_{13} \\
A_{23} & A_{d 23} & B_{23} \\
L_{3} & C_{3} & D_{3}
\end{array}\right]=\left[\begin{array}{ccccc}
0.1 & -0.2 & 0.3 & 0 & 0.1 \\
-0.2 & 0.1 & 0 & 0.1 & 0.25 \\
0.25 & 0.1 & 0.1 & 0 & 0 \\
0.05 & 0.3 & 0 & 0.1 & -0.28 \\
1 & 0.5 & 0.3 & 0.2 & 1 \\
0 & 0.3 & 1 & -0.3 & 0.3
\end{array}\right],} \\
& {\left[\begin{array}{ccc}
A_{14} & A_{d 14} & B_{14} \\
A_{24} & A_{d 24} & B_{24} \\
L_{4} & C_{4} & D_{4}
\end{array}\right]=}
\end{aligned}
$$

Four different cases for the transition probability matrix (TPM) are given in Table 1, where the transition probabilities (TPs) labeled with " "” and " " represent the unknown and uncertain elements, respectively. Specifically, Case 1, Case 2, Case 3, and Case 4 stand for the completely known TPs, deficient statistics of mode information (including known, partially unknown and uncertain TPS), partially unknown TPs, and completely unknown TPs, respectively.

For Case 2, it is assumed that the uncertain TPs comprise three vertices $\Pi_{s}, s=1,2,3$, where the third rows $\Pi_{s(3)}, S=1,2,3$, are given by

$$
\begin{aligned}
& \Pi_{1(3)}=\left[\begin{array}{llll}
\hat{\pi}_{31} & 0.2 & \hat{\pi}_{33} & 0.4
\end{array}\right], \\
& \Pi_{2(3)}=\left[\begin{array}{llll}
\hat{\pi}_{31} & 0.5 & \hat{\pi}_{33} & 0.3
\end{array}\right], \\
& \Pi_{3(3)}=\left[\begin{array}{llll}
\hat{\pi}_{31} & 0.3 & \hat{\pi}_{33} & 0.1
\end{array}\right],
\end{aligned}
$$

and the other rows in the three vertices are given with the same elements, that is,

$$
\begin{aligned}
\Pi_{s(1)} & =\left[\begin{array}{llll}
0.3 & 0.2 & 0.1 & 0.4
\end{array}\right], \\
\Pi_{s(2)} & =\left[\begin{array}{llll}
\hat{\pi}_{21} & \hat{\pi}_{22} & 0.3 & 0.2
\end{array}\right], \\
\Pi_{s(4)} & =\left[\begin{array}{llll}
0.2 & \hat{\pi}_{42} & \hat{\pi}_{43} & \hat{\pi}_{44}
\end{array}\right], \quad s=1,2,3 .
\end{aligned}
$$

Our purpose here is to design a filter of the form (5) for the above system such that the 2-D filtering error system is stochastically stable with an $\mathcal{H}_{\infty}$ performance $\gamma$. A detailed comparison between the minimum $\mathcal{H}_{\infty}$ performance indices $\gamma_{\text {min }}$ obtained based on Theorems 1 and 2 is listed in Table 2 . The results clearly show the advantages of Theorem 1 over

Table 1

Four different TPMs.

\begin{tabular}{ll}
\hline Case 1: Completely known TPM & Case 2: Deficient TPM1 \\
{$\left[\begin{array}{llll}0.3 & 0.2 & 0.1 & 0.4 \\
0.3 & 0.2 & 0.3 & 0.2 \\
0.1 & 0.5 & 0.3 & 0.1 \\
0.2 & 0.2 & 0.1 & 0.5\end{array}\right]$} & {$\left[\begin{array}{llll}0.3 & 0.2 & 0.1 & 0.4 \\
\hat{\pi}_{21} & \hat{\pi}_{22} & 0.3 & 0.2 \\
\hat{\pi}_{31} & \tilde{\pi}_{32} & \hat{\pi}_{33} & \tilde{\pi}_{34} \\
0.2 & \hat{\pi}_{42} & \hat{\pi}_{43} & \hat{\pi}_{44}\end{array}\right]$} \\
Case 3: Deficient TPM2 & Case 4: Completely unknown TPM \\
{$\left[\begin{array}{llll}0.3 & 0.2 & 0.1 & 0.4 \\
\hat{\pi}_{21} & \hat{\pi}_{22} & 0.3 & 0.2 \\
\hat{\pi}_{31} & \hat{\pi}_{32} & \hat{\pi}_{33} & \hat{\pi}_{34} \\
0.2 & \hat{\pi}_{42} & \hat{\pi}_{43} & \hat{\pi}_{44}\end{array}\right]$} & {$\left[\begin{array}{llll}\hat{\pi}_{11} & \hat{\pi}_{12} & \hat{\pi}_{13} & \hat{\pi}_{14} \\
\hat{\pi}_{21} & \hat{\pi}_{22} & \hat{\pi}_{23} & \hat{\pi}_{24} \\
\hat{\pi}_{31} & \hat{\pi}_{32} & \hat{\pi}_{33} & \hat{\pi}_{34} \\
\hat{\pi}_{41} & \hat{\pi}_{42} & \hat{\pi}_{43} & \hat{\pi}_{44}\end{array}\right]$} \\
\hline
\end{tabular}


Theorem 2 presented in this paper. Meantime, by inspection of Tables 1 and 2, it is easy to see that the more information on TPs is available, the better $\mathcal{H}_{\infty}$ performance can be obtained, which is effective to reduce the conservatism of filtering design. Therefore, the introduction of the uncertain TPs is significant.

Specifically, considering Case 2 shown in Table 1 and by applying Theorem 1, the feasible solutions of $\gamma_{\min }=0.3725$ for the full-order $\left(n_{f}=2\right)$ filter and $\gamma_{\min }=0.8165$ for the reduced-order $\left(n_{f}=1\right)$ filter are obtained respectively, and the corresponding filter gains are given by,

$$
\begin{aligned}
& {\left[\begin{array}{c|c|c}
A_{f 11} & A_{f 21} & B_{f 11} \\
\hline B_{f 21} & C_{f 1} & D_{f 1}
\end{array}\right]=\left[\begin{array}{cc|cc|cc}
0.2907 & -0.5806 & 0.1148 & -0.2178 & 1.0069 & -0.9991 \\
0.4310 & -0.8608 & 0.1837 & -0.3431 & 1.9188 & -1.6523 \\
\hline 0.7502 & -0.6785 & 0.3468 & -0.7481 & -0.9118 & 0.2625 \\
1.3746 & -1.3233 & -0.2774 & 0.5985 & -0.0706 & -0.2100
\end{array}\right],} \\
& {\left[\begin{array}{c|c|c}
A_{f 12} & A_{f 22} & B_{f 12} \\
\hline B_{f 22} & C_{f 2} & D_{f 2}
\end{array}\right]=\left[\begin{array}{cc|cc|cc}
0.0578 & -0.0563 & -0.0478 & 0.0322 & 0.4004 & -0.4400 \\
-0.2052 & 0.2001 & -0.0068 & 0.0049 & 1.0568 & -1.0386 \\
\hline 1.2995 & -1.0256 & 1.1492 & -1.1168 & -1.5547 & -0.0643 \\
0.0308 & -0.1820 & -0.1419 & 0.1379 & -0.7340 & 0.0079
\end{array}\right],} \\
& {\left[\begin{array}{c|c|c}
A_{f 13} & A_{f 23} & B_{f 13} \\
\hline B_{f 23} & C_{f 3} & D_{f 3}
\end{array}\right]=\left[\begin{array}{cc|cc|cc}
0.0440 & -0.2385 & -0.0224 & 0.3327 & -0.0526 & -0.0746 \\
0.0118 & -0.0642 & -0.0224 & 0.3331 & -0.3249 & 0.3102 \\
\hline 0.0106 & -0.2296 & 0.1683 & -0.9299 & -0.3499 & 1.2851 \\
0.3014 & -0.1682 & 0.0564 & -0.3115 & -0.0068 & 0.0624
\end{array}\right] \text {, }} \\
& {\left[\begin{array}{c|c|c}
A_{f 14} & A_{f 24} & B_{f 14} \\
\hline B_{f 24} & C_{f 4} & D_{f 4}
\end{array}\right]=\left[\begin{array}{cc|cc|cc}
0.2470 & -0.1912 & -0.0162 & 0.0552 & -0.0277 & -0.0233 \\
0.0232 & -0.0180 & -0.0167 & 0.0570 & 0.5089 & -0.6281 \\
\hline 0.0590 & -0.2520 & 0.0251 & -0.0203 & -0.2573 & -0.0700 \\
0.3160 & -0.6959 & 0.0384 & -0.0312 & -0.0613 & 0.2259
\end{array}\right],}
\end{aligned}
$$

for the full-order filter and

$$
\begin{aligned}
& {\left[\begin{array}{c|c|c}
A_{f 11} & A_{f 21} & B_{f 11} \\
\hline B_{f 21}^{\mathrm{T}} & C_{f 1} & D_{f 1}
\end{array}\right]=\left[\begin{array}{c|c|cc}
0.0489 & -0.0360 & 0.0670 & -0.2345 \\
\hline 1.2361 & 0.0725 & -2.0398 & 1.1727 \\
-0.8066 & -0.0580 & 0.8319 & -0.9382
\end{array}\right],} \\
& {\left[\begin{array}{c|c|c}
A_{f 12} & A_{f 22} & B_{f 12} \\
\hline B_{f 22}^{\mathrm{T}} & C_{f 2} & D_{f 2}
\end{array}\right]=\left[\begin{array}{c|c|cc}
0.0244 & 0.0418 & 0.0871 & -0.2716 \\
\hline 1.1955 & 0.7964 & -0.0779 & -0.2383 \\
-0.9380 & -0.0983 & -0.9163 & 0.0294
\end{array}\right] \text {, }} \\
& {\left[\begin{array}{c|c|c}
A_{f 13} & A_{f 23} & B_{f 13} \\
\hline B_{f 23}^{\mathrm{T}} & C_{f 3} & D_{f 3}
\end{array}\right]=\left[\begin{array}{c|c|cc}
-0.1928 & -0.0227 & -0.1702 & -0.1229 \\
\hline-0.0839 & -0.5695 & 0.1941 & 0.4818 \\
-0.0645 & -0.1908 & 0.1754 & -0.2067
\end{array}\right],} \\
& {\left[\begin{array}{c|c|c}
A_{f 14} & A_{f 24} & B_{f 14} \\
\hline B_{f 24}^{\mathrm{T}} & C_{f 4} & D_{f 4}
\end{array}\right]=\left[\begin{array}{c|c|cc}
0.0016 & 0.0001 & -0.1959 & 0.1095 \\
\hline-0.0295 & -0.0097 & -0.2792 & -0.0460 \\
-0.2449 & -0.0149 & -0.0948 & 0.2629
\end{array}\right],}
\end{aligned}
$$

for the reduced-order filter, respectively.

In order to further illustrate the effectiveness of the designed $\mathcal{H}_{\infty}$ filters, we present the simulation results with the above solutions. Let the boundary conditions be

Table 2

Comparison of minimum $H_{\infty}$ performance for different TPMs.

\begin{tabular}{lllll}
\hline \multirow{2}{*}{ TPMs } & Theorem 1 & & Theorem 2 \\
\cline { 2 - 3 } & Full-order & Reduced-order & Full-order & Reduced-order \\
\hline Case 1 & 0.2842 & 0.7518 & 0.6072 & 1.1084 \\
Case 2 & 0.3725 & 0.8165 & 0.8673 & 1.5292 \\
Case 3 & 0.3920 & 0.9077 & 1.1952 & 1.7297 \\
Case 4 & 0.4877 & 1.0604 & 1.8212 & 2.3384 \\
\hline
\end{tabular}




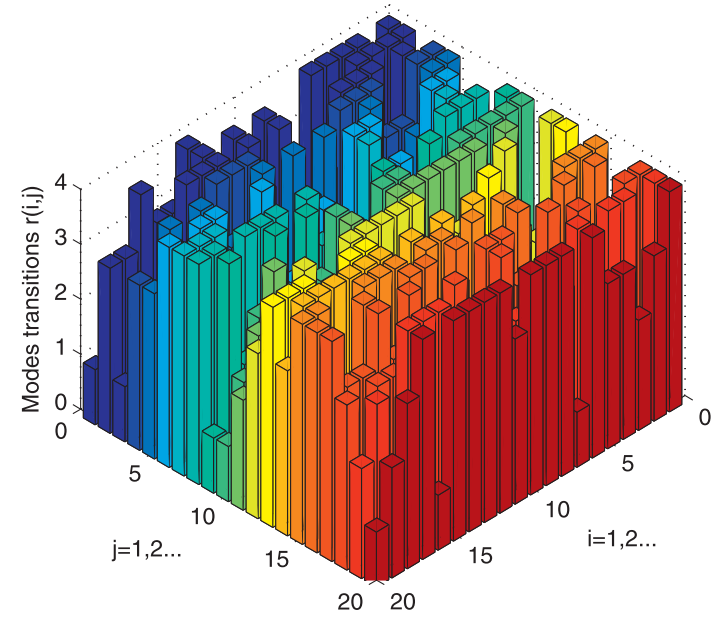

Fig. 1. One possible system mode evolution.

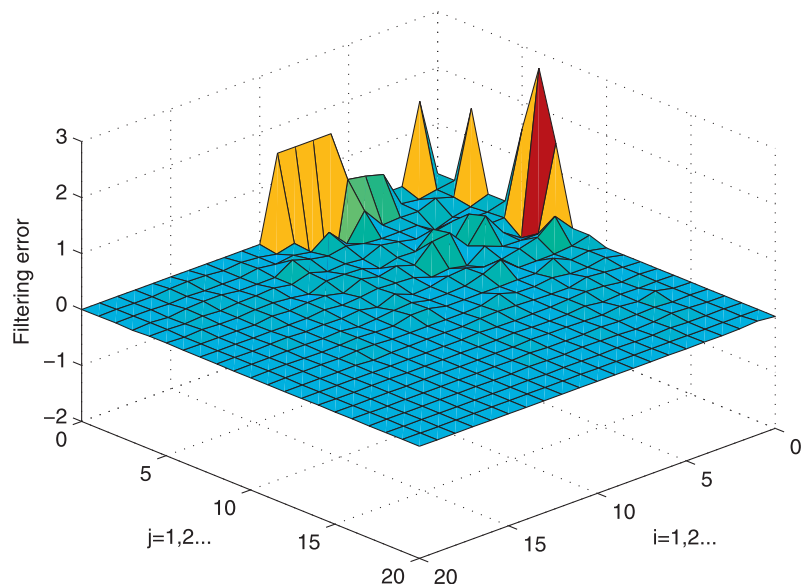

Fig. 2. Responses of the filtering error $\bar{z}(i, j)$ for the full-order case: the 1 st component.

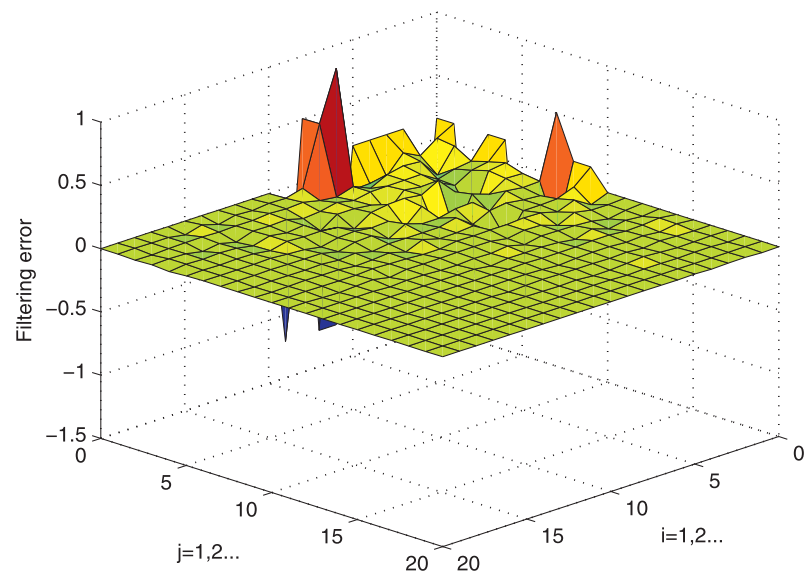

Fig. 3. Responses of the filtering error $\bar{z}(i, j)$ for the full-order case: the 2 nd component. 


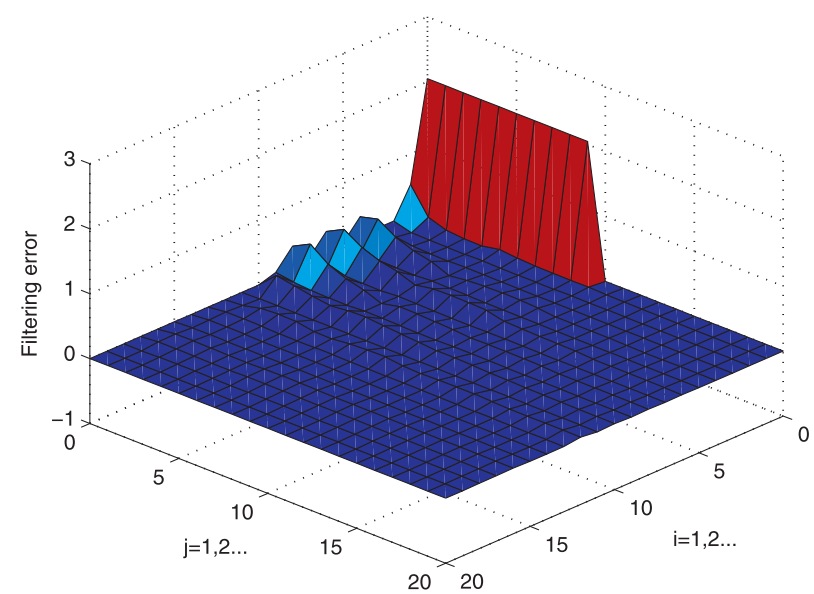

Fig. 4. Responses of the filtering error $\bar{z}(i, j)$ for the reduced-order case: the 1 st component.

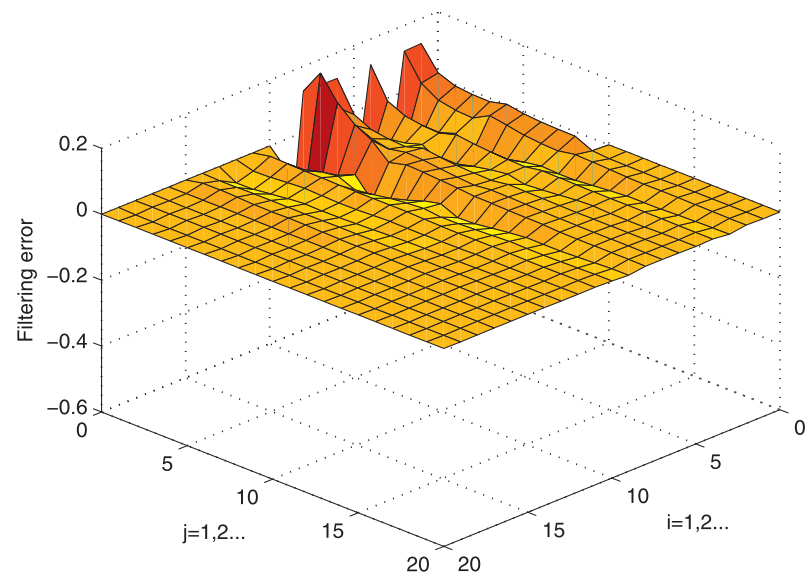

Fig. 5. Responses of the filtering error $\bar{z}(i, j)$ for the reduced-order case: the 2 nd component.

$$
x(t, i)=x(i, t)= \begin{cases}{\left[\begin{array}{ll}
-1 & 1.4
\end{array}\right]^{\mathrm{T}},} & 0 \leqslant i \leqslant 10, \\
{\left[\begin{array}{ll}
0 & 0
\end{array}\right]^{\mathrm{T}},} & i>10,\end{cases}
$$

where $-4 \leqslant t \leqslant 0$, and choose the delays $d_{1}=4$ (vertical direction), $d_{2}=4$ (horizontal direction), and disturbance input $w(i, j)$ as

$$
w(i, j)= \begin{cases}0.2, & 0 \leqslant i, j \leqslant 10 \\ 0, & \text { otherwise }\end{cases}
$$

With one possible realisation of the Markovian jumping mode shown in Fig. 1, the time responses of filtering errors are given in Figs. 2-5, respectively. It can be clearly observed from the simulation curves that, despite the deficient TPs, the filtering errors for both full-order and reduced-order filters converge to zero under the above conditions.

\section{Conclusions}

This paper has investigated the problem of $\mathcal{H}_{\infty}$ filtering for a class of two-dimensional (2-D) Markovian jump linear systems (MJLSs) with state-delays and deficient mode information. Such deficient mode information simultaneously involves the exactly known, partially unknown and polytopic-type uncertain transition probabilities, which are more general and practical. By the properties of the transition probability matrix (TPM) together with the convexification of uncertain domains, a new bounded real lemma for the filtering error system is firstly derived. Via some linearisation procedures, two methods have been proposed for the design of full-order and reduced-order filters. A simulation example has been given to illustrate effectiveness of the proposed approaches. 
Finally, it is worth mentioning that the $\mathcal{H}_{\infty}$ filtering design results presented in this paper are actually delay-independent, which do not include any information on the size of delays (i.e., the time-delays are allowed to be arbitrarily large). Extension of the current results to the delay-dependent case is interesting and expected to be less conservative, which deservers further investigation. Applications of the proposed results to some real-world complex 2-D systems such as the image data processing and transmission [30], thermal processes [32], gas absorption [33], and water stream heating [14], are also part of our future works.

\section{Acknowledgements}

The authors are grateful to the Editor-in-Chief, the Associate Editor, and anonymous reviewers for their constructive comments based on which the presentation of this paper has been greatly improved.

\section{References}

[1] W. Assawinchaichote, S.K. Nguang, P. Shi, Robust $\mathcal{H}_{\infty}$ fuzzy filter design for uncertain nonlinear singularly perturbed systems with Markovian jumps: an LMI approach, Inform. Sci. 177 (7) (2007) 1699-1714.

[2] E.K. Boukas, Stochastic Switching Systems: Analysis and Design, Birkhauser, Boston, 2005.

[3] S. Boyd, L. El Ghaoui, E. Feron, V. Balakrishnan, Linear Matrix Inequality in Systems and Control Theory, SIAM, Philadelphia, PA, 1994.

[4] B. Chen, H. Li, P. Shi, C. Lin, Q. Zhou, Delay-dependent stability analysis and controller synthesis for Markovian jump systems with state and input delays, Inform. Sci. 179 (16) (2009) 2851-2860.

[5] B. Chu, Y. Liu, On the asymptotic stability of positive 2-D systems described by the Roesser model, IEEE Trans. Circ. Syst. - Part II: Express Briefs 54 (12) (2007) 1102-1104.

[6] C. Du, L. Xie, C. Zhang, $\mathcal{H}_{\infty}$ control and robust stabilization of two-dimensional systems in Roesser models, Automatica 37 (2) (2001) $205-211$.

[7] D. Ding, X. Li, Y. Yin, X. Xie, Further studies on relaxed stabilization conditions for discrete-time two-dimension Takagi-Sugeno fuzzy systems, Inform. Sci. 189 (2012) $143-154$

[8] H. Gao, J. Lam, S. Xu, C. Wang, Stabilization and $\mathcal{H}_{\infty}$ control of two-dimensional Markovian jump systems, IMA J. Math. Control Inform. 21 (4) (2004) 377-392.

[9] A. Hmamed, C.E. Kasri, E.H. Tissir, T. Alvarez, F. Tadeo, Robust $\mathcal{H}_{\infty}$ filtering for uncertain 2-D continuous systems with delays, Int. J. Innovative Comput. Inform. Control 9 (5) (2013) 2167-2183.

[10] T. Hinamoto, Stability of 2-D discrete systems described by the Fornasini-Marchesini second model, IEEE Trans. Circ. Syst. - Part I: Regular Papers 44 (3) (1997) 254-257.

[11] H.R. Karimi, Robust delay-dependent $\mathcal{H}_{\infty}$ control of uncertain time-delay systems with mixed neutral, discrete, and distributed time-delays and Markovian switching parameters, IEEE Trans. Circ. Syst. - Part I: Regular Papers 58 (8) (2011) 1910-1923.

[12] M. Karan, P. Shi, C.Y. Kaya, Transition probability bounds for the stochastic stability robustness of continuous- and discrete-time Markovian jump linear systems, Automatica 42 (12) (2006) 2159-2168.

[13] N.N. Krasovskii, E.A. Lidskii, Analytical design of controllers in systems with random attributes, Autom. Remote Control Parts 1-3 22 (1) (1961) 10211025.

[14] T. Kaczorek, Two-Dimensional Linear Systems, Springer, Berlin, Germany, 1985.

[15] F. Li, X. Zhang, Delay-range-dependent robust $\mathcal{H}_{\infty}$ filtering for singular LPV systems with time variant delay, Int. J. Innovative Comput. Inform. Control 9 (1) (2013) 339-353.

[16] H. Liu, D.W.C. Ho, F. Sun, Design of $\mathcal{H}_{\infty}$ filter for Markov jumping linear systems with non-accessible mode information, Automatica 44 (10) (2008) $2655-2660$.

[17] W.S. Lu, On a Lyapunov approach to stability analysis of 2-D digital filters, IEEE Trans. Circ. Syst. - Part I: Regular Papers 41 (10) (1994) 665-669.

[18] W. Paszke, J. Lam, K. Galkowski, S. Xu, Z. Lin, Robust stability and stabilisation of 2D discrete state-delayed systems, Syst. Control Lett. 51 (3-4) (2004) 277-291.

[19] J. Qiu, G. Feng, J. Yang, A new design of delay-dependent robust $\mathcal{H}_{\infty}$ filtering for discrete-time T-S fuzzy systems with time-varying delay, IEEE Trans. Fuzzy Syst. 17 (5) (2009) 1044-1058.

[20] J. Qiu, G. Feng, J. Yang, Delay-dependent non-synchronized robust $\mathcal{H}_{\infty}$ state estimation for discrete-time piecewise linear delay systems, Int. J. Adaptive Control Signal Process. 23 (2) (2009) 1082-1096.

[21] J. Qiu, G. Feng, J. Yang, A new design of delay-dependent robust $\mathcal{H}_{\infty}$ filtering for continuous-time polytopic systems with time-varying delay, Int. J. Robust Nonlinear Control 20 (3)(2010) 346-365.

[22] X. Qiu, P. Shi, J. Xiao, J. Yang, A novel active filter for unbalanced 3-phase 4-wire power system based on linear adaptive notch filter and fuzzy adaptive hysteresis controller, Int. J. Innovative Comput. Inform. Control 9 (6) (2013) 2619-2634.

[23] R.P. Roesser, A discrete state-space model for linear image processing, IEEE Trans. Autom. Control 20 (1) (1975) 1-10.

[24] P. Shi, E.K. Boukas, R.K. Agarwal, Kalman filtering for continuous-time uncertain systems with Markovian jumping parameters, IEEE Trans. Autom. Control 44 (8) (1999) 1592-1597.

[25] P. Shi, M.S. Mahmoud, S.K. Nguang, A. Ismail, Robust filtering for jumping systems with mode-dependent delays, Signal Process. 86 (1) (2006) $140-152$.

[26] P. Shi, M.S. Mahmoud, J. Yi, A. Ismail, Worst case control of uncertain jumping systems with multi-state and input delay information, Inform. Sci. 176 (2) (2006) $186-200$.

[27] X. Su, P. Shi, L. Wu, S.K. Nguang, Induced $l_{2}$ filtering of fuzzy stochastic systems with time-varying delays, IEEE Trans. Cybernet. 43 (4) (2013) $1251-$ 1264.

[28] X. Su, P. Shi, L. Wu, Y. Song, A novel approach to filter design for T-S fuzzy discrete-time systems with time-varying delay, IEEE Trans. Fuzzy Syst. 20 (6) (2012) 1114-1129.

[29] X. Su, P. Shi, L. Wu, Y. Song, A novel control design on discrete-time Takagi-Sugeno fuzzy systems with time-varying delays, IEEE Trans. Fuzzy Syst. 21 (4) (2013) 655-671.

[30] H.D. Tuan, P. Apkarian, T.Q. Nguyen, T. Narikiyo, Robust mixed $\mathcal{H}_{2} / \mathcal{H}_{\infty}$ filtering of 2-D system, IEEE Trans. Signal Process. 50 (7) (2002) $1759-1771$.

[31] H. Wu, K. Cai, Robust fuzzy control for uncertain discrete-time nonlinear Markovian jump systems without mode observations, Inform. Sci. 177 (6) (2007) 1509-1522.

[32] L. Wu, P. Shi, H. Gao, C. Wang, $\mathcal{H}_{\infty}$ mode reduction for two-dimensional discrete state-delayed systems, IEE Proc.-Vision Image Signal Process. 153 (6) (2006) 769-784.

[33] L. Wu, P. Shi, H. Gao, C. Wang, $\mathcal{H}_{\infty}$ filtering for 2-D Markovian jump systems, Automatica 44 (7) (2008) 1849-1858.

[34] L. Wu, X. Su, P. Shi, Output feedback control of Markovian jump repeated scalar nonlinear systems, IEEE Trans. Autom. Control (2013), http:// dx.doi.org/10.1109/TAC.2013.2267353.

[35] L. Wu, Z. Wang, H. Gao, C. Wang, Filtering for uncertain 2-D discrete systems with state delays, Signal Process. 87 (9) (2007) $2213-2230$.

[36] L. Wu, W. Zheng, H. Gao, Dissipativity-based sliding mode control of switched stochastic systems, IEEE Trans. Autom. Control 58 (3) (2013) 785-793. 
[37] Y. Wei, J. Qiu, H.R. Karimi, M. Wang, A new design of $\mathcal{H}_{\infty}$ filtering for continuous-time Markovian jump systems with time-varying delay and partially accessible mode information, Signal Process. 93 (9) (2013) 2392-2407.

[38] Z. Wu, P. Shi, H. Su, J. Chu, $l_{2}-l_{\infty}$ filter design for discrete-time singular Markovian jump systems with time-varying delays, Inform. Sci. 181 (24) (2011) 5534-5547.

[39] J. Xiong, J. Lam, Fixed-order robust $\mathcal{H}_{\infty}$ filter design for Markovian jump systems with uncertain switching probabilities, IEEE Trans. Signal Process. 54 (4) (2006) 1421-1430.

[40] H. Xu, Y. Zou, S. Xu, J. Lam, Q. Wang, $\mathcal{H}_{\infty}$ model reduction of 2-D singular Roesser models, Multidim. Syst. Signal Process. 16 (3) (2005) $285-304$.

[41] S. Xu, J. Lam, Z. Lin, K. Galkowski, W. Paszke, B. Sulikowski, E. Rogers, D. Owens, Positive real control of two-dimensional systems: Roesser models and linear repetitive processes, Int. J. Control 76 (11) (2003) 1047-1058.

[42] S. Xu, J. Lam, X. Mao, Delay-dependent $\mathcal{H}_{\infty}$ control and filtering for uncertain Markovian jump systems with time-varying delays, IEEE Trans. Circ. Syst. - Part I: Regular Papers 54 (9) (2007) 2070-2077.

[43] L. Zhang, E.K. Boukas, Mode-dependent $\mathcal{H}_{\infty}$ filtering for discrete-time Markovian jump linear systems with partly unknown transition probabilities, Automatica 45 (6) (2009) 1462-1467.

[44] Y. Zhang, Y. He, M. Wu, J. Zhang, Stabilization for Markovian jump systems with partial information on transition probability based on free-connection weighting matrices, Automatica 47 (1) (2011) 79-84. 